\title{
Traduire
}

Ine autre perspective sur I t tadaduction

Revue française de la traduction

$219 \mid 2008$

Le temps de la réflexion

\section{Traduire la publicité ou comment justifier et argumenter ses choix de traduction}

\section{Mathieu Guidère}

\section{(2) OpenEdition}

12 Journals

Édition électronique

URL : http://journals.openedition.org/traduire/875

DOI : $10.4000 /$ traduire.875

ISSN : 2272-9992

Éditeur

Société française des traducteurs

Édition imprimée

Date de publication : 15 décembre 2008

Pagination : 22-50

ISSN : 0395-773X

\section{Référence électronique}

Mathieu Guidère, «Traduire la publicité ou comment justifier et argumenter ses choix de traduction »,

Traduire [En ligne], 219 | 2008, mis en ligne le 15 décembre 2008, consulté le 21 décembre 2020. URL : http://journals.openedition.org/traduire/875 ; DOI : https://doi.org/10.4000/traduire.875 


\section{Traduire la publicité \\ ou \\ comment justifier et argumenter ses choix de traduction}

\section{Mathieu Guidère}

Tous ceux qui se sont essayés au domaine de la traduction publicitaire savent que le chemin est semé d'embûches et que la tâche est loin d'être aisée, principalement en raison des enjeux économiques et financiers liés à la publicité. Malgré la diversité des modes d'exercice de la profession, on en revient toujours au même problème : plus qu'ailleurs, le traducteur est souvent amené à argumenter ses décisions et à justifier ses choix de traduction.

Il faut dire que la nature même des textes à traduire se prête bien à la multiplicité des interprétations et des solutions traductionnelles. Par sa conception même, la publicité est d'emblée un message complexe qui peut recevoir plusieurs traductions et qui se situe au croisement de plusieurs spécialités dont l'éclairage est à prendre en considération. Aussi, lorsque le traducteur propose une solution particulière, se trouvet-il toujours quelqu'un pour contester la traduction proposée ou pour suggérer une meilleure façon de formuler ou d'adapter le message publicitaire.

C'est que la confusion règne : traduire la publicité est tantôt classé parmi les formes de la traduction spécialisée, tantôt parmi les formes de rédaction ou de communication. En fonction des interlocuteurs, on se trouve confronté à une logique de transfert linguistique ou bien d'adaptation culturelle. Tout dépend de la conception qui sous-tend le travail entrepris et des stratégies de communication définies par le 22 commanditaire (standardisation, localisation, adaptation, etc.). 
Cet enchevêtrement des facteurs et des situations conduit le traducteur à prévenir en amont tout risque de "contestation » de son travail en réalisant lui-même une traduction argumentée visant à parer les objections éventuelles concernant ses choix de traduction. L'objet de cet article est d'exposer une méthode d'argumentation traductologique qui a fait ses preuves aussi bien dans le milieu des professionnels de la publicité que dans la formation universitaire à la traduction spécialisée. Elle concerne exclusivement le texte publicitaire, c'est-à-dire le rédactionnel. Les autres parties de l'annonce, et notamment la relation entre le texte et l'image, ont fait l'objet d'études exhaustives auxquelles nous renvoyons le lecteur (voir la bibliographie).

\section{Qu'est-ce que la traduction argumentée ?}

Largumentation est l'action de justifier ses positions et ses propositions, en l'occurrence ses solutions de traduction. Argumenter signifie littéralement : «présenter des arguments, prouver, persuader » et, du point de vue logique, un argument n'est rien d'autre qu'un " raisonnement destiné à prouver ou à réfuter une proposition et, par extension, une preuve à l'appui ou à l'encontre d'une proposition " (Le Robert).

La traduction argumentée est, par conséquent, le fait de démontrer par des arguments la validité ou l'inadéquation d'une traduction personnelle ou bien d'une équivalence spécifique. Cela signifie concrètement :

1) Fournir des arguments à l'appui d'un choix lexical ou terminologique.

2) Avancer des exemples pour convaincre d'un choix syntaxique ou stylistique.

3) Réfuter les arguments avancés contre une traduction ou une adaptation.

5) Documenter ses choix de traduction par des recherches précises et ciblées. 
Il existe ainsi un double processus de justification dans la traduction argumentée : «offensif " d'un côté (argumenter) et " défensif » de l'autre (contre-argumenter).

L'auto-argumentation consiste à fournir des arguments pour démontrer la justesse ou la pertinence de sa propre traduction. En d'autres termes, il s'agit de traduire soi-même un texte publicitaire puis de justifier ses choix et ses décisions au niveau des mots, des expressions et des phrases.

À l'inverse, la contre-argumentation consiste à réfuter, par des arguments valides, une solution proposée par d'autres personnes ou bien une traduction réalisée par des non-professionnels sur la foi de leurs connaissances linguistiques. Il s'agit donc d'une critique raisonnée de la traduction d'autrui et appuyée par des contre-propositions considérées comme plus pertinentes ou plus adéquates au contexte.

La pertinence des arguments ou des contre-arguments avancés dépend de leur nature (argument lexical, stylistique, culturel...), car tous les arguments ne se valent pas du point de vue de leur force de persuasion (argument consensuel, convaincant, décisif, etc.).

Selon les publicités et les types de discours, le traducteur peut être amené à argumenter l'un des aspects suivants :

1) Argumenter la compréhension, c'est-à-dire préciser un aspect du sens qui pose problème dans la manière de comprendre le texte de départ (ou texte source). Cela se fait par le recours au contexte et, accessoirement au dictionnaire, pour justifier une certaine façon de lire et d'interpréter le sens original.

2) Argumenter la réexpression, c'est-à-dire justifier un choix stylistique adopté lors de la rédaction du texte d'arrivée (ou texte cible). Cela se fait par le biais de la stylistique en expliquant chaque choix de reformulation des segments ou des phrases de telle ou telle manière.

3) Argumenter l'équivalence, c'est-à-dire expliquer tout ce qui pose problème dans la recherche et la sélection des correspondances lexi- 
cales et syntaxiques. Cela se fait par l'intermédiaire de la recherche documentaire et/ou terminologique qui permet de valider un choix d'équivalence par rapport à un autre.

\section{Comment procéder pour l'argumentation ?}

Argumenter une traduction publicitaire passe par plusieurs étapes que nous présentons sommairement ci-après, avant d'en donner des exemples précis de cas pratiques.

1) Contextualiser le texte publicitaire : type de support, date de production, objet du message, origine de la commande, marque concernée, logo international et local, cible commerciale visée, etc.

2) Identifier les difficultés dans le texte de départ : lecture et détection des difficultés lexicales, syntaxiques, stylistiques et, surtout, culturelles, c'est-à-dire des difficultés pragmatiques qui pourraient freiner la compréhension du sens d'un mot, d'une expression, d'une phrase ou encore d'une référence littéraire ou historique. Dans un contexte didactique, ces difficultés peuvent être classées dans une catégorie précise, de préférence sous forme de tableau, comme suit :

\begin{tabular}{|c|c|c|}
\hline $\begin{array}{l}\text { Difficultés lexicales ou } \\
\text { choix terminologiques } \\
\text { de l'original (TS) }\end{array}$ & $\begin{array}{c}\text { Difficultés syntaxiques } \\
\text { ou choix stylistiques } \\
\text { de l'original (TS) }\end{array}$ & $\begin{array}{c}\text { Difficultés culturelles } \\
\text { ou choix pragmatiques } \\
\text { de l'original (TS) }\end{array}$ \\
\hline $\begin{array}{l}\text { - Je ne comprends pas le } \\
\text { sens précis du mot ou } \\
\text { du terme malgré le } \\
\text { contexte verbal. } \\
\text { - Je reconnais un champ } \\
\text { lexical ou sémantique } \\
\text { particulier qui parcourt } \\
\text { le texte d'origine. }\end{array}$ & $\begin{array}{l}\text { - Je ne comprends pas } \\
\text { l'expression ou la formu- } \\
\text { lation originale. } \\
\text { - Je m'interroge sur la } \\
\text { structure ou sur la } \\
\text { construction en langue } \\
\text { source. } \\
\text { - Je reconnais un choix sty- } \\
\text { listique particulier dans } \\
\text { le texte de départ (figure } \\
\text { de style, trope, etc.). }\end{array}$ & $\begin{array}{l}\text { - Je ne connais pas la } \\
\text { référence littéraire ou la } \\
\text { notion culturelle citée } \\
\text { dans le texte. } \\
\text { - Je détecte un signe ou } \\
\text { un symbole typique de } \\
\text { la culture source. }\end{array}$ \\
\hline
\end{tabular}


Voici quelques exemples de difficultés ou de choix stylistiques fréquents dans les textes publicitaires concernant des produits de beauté (EN/FR) :

\begin{tabular}{|l|c|c|}
\hline \multicolumn{1}{|c|}{ Difficultés lexicales } & Difficultés stylistiques & Difficultés culturelles \\
\hline - Mots polysémiques & - Grammaire inhabituelle & - Fait de société peu connu \\
- Termes absents du dic- & en langue source & - Référence poétique \\
tionnaire & $-\begin{array}{c}\text { Phrases alambiquées ou } \\
\text { constructions ambiguës }\end{array}$ & - Jeu de mots \\
- Mots composés pour & Expressions idiomatiques & - Allusion historique \\
l'occasion & difficiles à transposer & - Intertextualité \\
- Néologismes & Choix rhétoriques : pa- & - Implicite discursif \\
& rallélismes, métaphorisa- & \\
& tion, personnalisation, & \\
& etc. & \\
\hline
\end{tabular}

3) Rechercher une solution à chacune des difficultés notées dans le texte de départ (TS) :

- En ayant recours au dictionnaire unilingue (pour le sens des mots ou des termes);

- En ayant recours à une grammaire de la langue source pour les constructions phrastiques ;

- En ayant recours à des corpus de textes disponibles sur Internet pour les formules typiques, les expressions idiomatiques ou encore les innovations terminologiques ;

- En ayant recours à des dictionnaires encyclopédiques pour les faits de culture ou encore pour les références littéraires et artistiques.

Pour éviter les contestations inutiles de la part du commanditaire, il est important que l'outil employé à chaque fois soit précisément en relation avec la difficulté observée ou le choix sémiostylistique mis en évidence : par exemple, on utilise un dictionnaire unilingue pour une difficulté lexicale du texte de départ, mais un dictionnaire encyclopé26 dique pour cerner une référence culturelle. 
4) Justifier l'équivalence proposée à partir de cette recherche, c'est-àdire la manière de résoudre le problème. Il s'agit, en d'autres termes, d'expliquer - de façon claire et précise - le processus de traduction suivi, en offrant des explications d'ordre sémantique, référentiel, contextuel ou culturel.

5) Exposer les possibilités d'expression envisageables et justifier la reformulation ou la phrase choisie pour la version traduite :

Exemple didactique : "Pour traduire cette phrase, il est possible d'envisager plusieurs formulations en français... (citer les possibilités d'expression)... En raison du contexte (préciser) et des choix lexicaux de l'original (préciser), nous préférons la deuxième solution citée parce que... Si la solution retenue est ... alors le lecteur comprendra... On pourrait également traduire par... mais dans ce cas, la connotation du message serait... parce que ce mot / cette expression implique dans la langue source l'idée de... Par conséquent, il est préférable de traduire par... puisque l'objectif dans le texte original est de positiver le produit / valoriser le consommateur / mettre en avant la marque..., etc. ».

\section{Étude de cas : traduction argumentée d'un texte publicitaire}

Texte d'appui : L'Oréal, Preference, Les Rouges Romantiques

\begin{tabular}{|l|l|}
\hline \multicolumn{1}{|c|}{ Version anglaise } & \multicolumn{1}{c|}{ Version française } \\
\hline$N E W$ & INNOVATION \\
Preference & Preference $\circledast$ \\
les & les \\
Rouges & Rouges \\
Romantiques & Romantiques \\
Three new passionate and intense red & Nos trois nouvelles nuances d'un rouge \\
shades you'll adore! A patented stay-true & passion aux reflets intenses, vous allez
\end{tabular}




\begin{tabular}{|c|c|}
\hline $\begin{array}{l}\text { colorant means color won't fade out, and } \\
\text { a special conditioner with a UV filter } \\
\text { means hair won't dry out. }\end{array}$ & $\begin{array}{l}\text { les adorer ! Les Rouges Romantiques* } \\
\text { vous garantissent une couleur authen- } \\
\text { tique qui ne s'estompera pas et un soin } \\
\text { unique anti-UV qui ne dessèchera pas } \\
\text { vos cheveux. }\end{array}$ \\
\hline $\begin{array}{l}\text { If you want to fall head over heels in love } \\
\text { with your red, try Les Rouges } \\
\text { Romantiques by Preference. }\end{array}$ & $\begin{array}{l}\text { Vivez votre passion en rouge, essayez les } \\
\text { Rouges Romantiques de Preference. }\end{array}$ \\
\hline Because You're Worth It ${ }^{\circledR}$ & Parce que je le vaux bien ${ }^{\circledR}$ \\
\hline L'Oréal & L'Oréal \\
\hline Paris & Paris \\
\hline
\end{tabular}

\section{1) Contextualisation de la publicité (fiche descriptive) :}

- Le texte proposé à la traduction est un texte de type publicitaire visant la promotion d'un produit pour les cheveux, dont la cible commerciale est principalement féminine.

- Iconographie : produit + prescripteur (ne change pas ici, à garder telle quelle).

- Charte graphique : couleur rouge dominante (à garder telle quelle, ne présente pas de difficulté de perception culturelle dans les deux langues concernées).

- Nom de la marque : L'Oréal (déposé en français comme en anglais, ne change pas, à garder tel quel).

- Nom de produit : Preference, gamme "Les Rouges Magiques " (déposé, à garder tel quel).

- Nom de caution : celui qui recommande le produit, aucun ici (si présent sur l'original, à transcrire).

- Nom d'origine : Paris (argument commercial lié à l'image de marque, à transcrire dans la langue cible si différents : ex. en italien, en arabe, etc.).

* Formule brevetée. 
- Phrase d'accroche (head line) : aucune ici.

- Phrase d'assise (base line) : aucune ici.

- Slogan de la marque (déposé dans les deux langues) ; choix entre deux possibilités de traduction qui correspondent à deux axes de communication :

1) "Parce que vous le valez bien", Because you're worth it : traduction littérale, adresse directe au consommateur ; axe de communication : valorisation de l'interlocuteur et projection sur la marque.

2) "Parce que je le vaux bien", Because I'm worth it : traduction inacceptable en anglais car connotation de prétention ; c'est le modèle de la marque qui parle ; adresse indirecte au consommateur ; axe de communication différent : auto-valorisation et identification au locuteur.

$\rightarrow$ Pour le public francophone, le slogan («Parce que je le vaux bien ») est entré dans les mœurs alors que pour le public anglophone, le slogan n'est pas encore idiomatique.

\section{2) Identification des difficultés en TS (fiche diagnostic)}

\begin{tabular}{|c|c|c|}
\hline $\begin{array}{l}\text { Difficultés lexicales } \\
\text { ou choix sémantiques }\end{array}$ & $\begin{array}{l}\text { Difficultés } \\
\text { ou choix } s\end{array}$ & $\begin{array}{l}\text { Difficultés culturelles } \\
\text { ou choix pragmatiques }\end{array}$ \\
\hline $\begin{array}{l}\text { - Shades } \\
\text { - Stay-true } \\
\text { - To fade out } \\
\text { - To dry out }\end{array}$ & $\begin{array}{l}\text { - Means... means... (paral- } \\
\text { lélisme à préserver dans } \\
\text { la version traduite } \\
\text { - Won't... won't... (double } \\
\text { négation à garder dans la } \\
\text { traduction) } \\
\text { - Fade out... dry out... } \\
\text { (parallélisme de structure } \\
\text { verbale) }\end{array}$ & $\begin{array}{l}\text { - To fall head over heels in } \\
\text { love } \\
\text { - Distinction entre le } \\
\text { nom de la gamme et le } \\
\text { nom du produit } \\
\text { - Typographie variable } \\
\text { (agrandissement de cer- } \\
\text { taines lettres) }\end{array}$ \\
\hline
\end{tabular}


3) Recherche d'une solution lexicographique (fiche lexicale)

Shades : ombre, nuance, ton, soupçon

- A shade: ombre; in the shade of a tree : à l'ombre/sous l'ombrage d'un arbre.

- Shade of colour/opinion : nuance $\mathrm{f}$, ton $\mathrm{m}$.

- A shade of vulgarity : un soupçon de vulgarité.

- To shade price : baisser ou diminuer progressivement les prix.

To fade out : passer, perdre son éclat, se décolorer, baisser, diminuer, s'effacer, diminuer, décliner, s'affaiblir, s'évanouir, disparaître, s'éteindre

- To fade out (colour) : passer, perdre son éclat, se décolorer, baisser, diminuer.

- Also fade away : s'effacer, diminuer, décliner, s'affaiblir, s'évanouir :

- the castle faded from sight : le château disparut aux regards ;

- her voice faded into silence : sa voix s'est éteinte ;

- to fade into the background (person) : se fondre dans le décor.

To dry out : dessécher, sécher, tenir au sec

- Her throat/mouth was dry: elle avait la gorge/la bouche sèche.

- At least you're in the warm and the dry: au moins vous êtes au chaud et au sec :

- dry away from direct heat (on label) : " ne pas sécher près d'une source de chaleur";

- to dry one's eyes or one's tears : sécher ses larmes ou ses pleurs.

4) Justification des équivalences choisies (fiche argumentaire)

1) Pour shades, la meilleure équivalence ici est "nuance " parce qu'il s'agit d'un produit de coloration pour les cheveux et qu'une " couleur " possède plusieurs " nuances", ce qui rend bien l'idée de " gamme " présente dans le nom du produit (Les Rouges Romantiques de Preference de L'Oréal). 
2) Pour stay-true, la traduction la plus adéquate ici serait " authentique " parce que cet adjectif indique à la fois la dimension temporelle, la durée (stay-) et l'idée de vérité (-true). En français, une chose " authentique " paraît naturelle et possède forcément une histoire. Le sens voulu ici est que cette coloration donne aux cheveux un aspect naturel qui ne s'affadit pas avec le temps mais garde son éclat comme s'il s'agissait d'une couleur naturelle justement.

3) Pour to fade out, à partir de la définition et des exemples donnés dans le Collins, je pense que le sens voulu est ... c'est pourquoi, je propose l'équivalent français suivant... En effet, le mot aurait ainsi les mêmes sèmes dans les deux langues... (Justification sémantique et référentielle).

4) Pour to dry out, à partir d'une recherche faite sur Internet (*référence du site web), j'ai trouvé l'explication suivante de l'expression/ de la notion... c'est pourquoi, je pense qu'il est possible de la traduire en français par... En effet, cette solution permet de garder la même référence historique / littéraire... (Justification contextuelle ou culturelle).

\section{5) Possibilités de réexpression (fiche reformulation)}

- Phrase de départ : Three new passionate and intense red shades you'll adore!

- Pour le segment : you'll adore, les possibilités de réexpression sont :

1) Vous allez aimer/apprécier...

2) Vous allez adorer/adopter...

3) Vous allez les adorer/vous adorerez, nos trois nouvelles nuances de rouge (mise en relief de l'utilisateur).

4) Nos trois nouvelles colorations de rouge intenses et passionnées, vous allez les adorer (mise en relief de la marque).

5) Trois nouvelles colorations de rouge, vous allez les adorer ! (Communication produit). 
- Pour le segment : passionate $(X)$ and intense $(Y)$ red $(Z)$ shades $(W)$ :

$$
X \quad \text { and } Y \quad Z \quad W
$$

En français, les traductions possibles, c'est-à-dire correctes et acceptables, sont les suivantes :

$Z X$ and $Y$ : "...d'un rouge passionné et intense ".

$Z \quad Y$ and $X:$ "...d'un rouge intense et passionné ».

ou bien :

$W \ldots X$ and $Y:$ "...aux reflets passionnés et intenses"

$W \ldots Y$ and $X:$ "...aux reflets intenses et passionnés ».

ou encore :

$Z X$ and $W Y:$ "... d'un rouge passionné et de reflets intenses". $Z Y$ and $W X:$ «... d'un rouge intense et de reflets passionnés".

Solution proposée : substantivation de "passionné " et suppression de la coordination (et) : «... d'un rouge passion aux reflets intenses ".

- Phrase 2 : Patented stay-true colorant means color won't fade out.

Possibilités de reformulation lexicale et syntaxique :

1) Une formule de coloration brevetée qui reste fidèle.

2) Une formule brevetée pour une couleur qui ne s'estompe pas.

3) Des pigments haute résistance brevetés vous garantissent un résultat éclatant.

4) Une formule brevetée vous garantira une tenue irréprochable de la couleur.

Solution proposée (recherche de la fluidité de l'expression) :

- Les Rouges Romantiques* vous garantissent une couleur authentique qui ne s'estompera pas.

$32 \quad$ * Formule brevetée. 
- Phrase 3 : and a special conditioner with a UV filter means hair won't dry out.

Possibilités de reformulation lexicale et syntaxique :

1) et un soin unique qui empêchera vos cheveux de se dessécher grâce à son filtre UV;

2) grâce à un soin spécial doté d'un filtre UV, vos cheveux sont protégés de tout dessèchement.

Solution proposée (conserve le parallélisme avec la première partie de la phrase) :

- Un soin unique anti-UV qui ne dessèchera pas vos cheveux.

Reformulation de la totalité de la phrase :

Les Rouges Romantiques* vous garantissent une couleur authentique qui ne s'estompera pas et un soin unique anti-UV qui ne dessèchera pas vos cheveux.

- Phrase 4: If you want to fall head over in love with red, try Les Rouges Romantiques by Preference.

Possibilités de reformulation lexicale et syntaxique :

1) Si vous voulez rougir de plaisir, essayez Les Rouges Romantiques de Preference.

2) $\mathrm{Si}$ vous voulez tomber raide dingue, laissez-vous tenter par Les Rouges Romantiques de Preference.

3) $\mathrm{Si}$ vous désirez ardemment un rouge passion, craquez pour Les Rouges Romantiques de Preference.

Solution proposée :

- Vivez votre passion en rouge, essayez Les Rouges Romantiques de Preference.

* Formule brevetée. 


\section{6) Commentaire argumenté (fiche rédaction)}

\section{Les choix du traducteur}

1) Au niveau sémantique, le traducteur procède à un choix du vocabulaire employé.

Par exemple, pour shades : nuances, teintes, reflets, tons,...

2) Au niveau syntaxique, le traducteur procède à un choix de point de vue particulier.

Par exemple, pour you'll adore : "Vous allez adorer " (en début de phrase) ou bien "Vous adorerez" (en fin de phrase).

3) Au niveau communicationnel, le traducteur procède au choix d'un axe (Com. Produit, Client, Marque).

Par exemple, pour la première phrase Three new... you'll adore, il peut mettre en relief différents aspects par le choix d'un type de formulation :

- "Trois colorations..." (Mise en avant du produit).

- "Vous allez adorer..." (Mise en avant du client).

- «Nos colorations..." (Mise en avant de la marque).

\section{Les décisions du traducteur}

1) Pour le lexique, la décision se fait par élimination selon l'adéquation au contexte :

- Three new... shades...

- Trois nouvelles nuances.../reflets/teintes/couleurs/colorations...

2) Pour la syntaxe, la décision se fait en référence à l'axe de communication (Com. Produit, Client, Marque) :

- Vous allez adorer nos trois nouvelles colorations... !

- Les trois nouvelles colorations... vous allez les adorer!

3) Pour la communication, la décision se fait par rapport au point de vue qu'on souhaite mettre en avant (Sujet, Objet, Interaction) : 
- Vous allez adorer... (Sujet).

- Trois nouvelles colorations... (Objet).

- Nos trois nouvelles colorations... (Interaction/implication du consommateur).

La créativité du traducteur

"La créativité, c'est la liberté dans la fidélité »(1).

Exemple : passionate $(X)$ and intense $(Y)$ red $(Z)$ shades $(W)$

$$
X \text { and } Y \quad Z \quad W
$$

$Z X$ and $Y:$ "...d'un rouge passionné et intense ".

$Z \quad Y$ and $X:$ "...d'un rouge intense et passionné ".

$W \ldots X$ and $Y:$ "...aux reflets passionnés et intenses".

$W \ldots Y$ and $X:$ "...aux reflets intenses et passionnés ».

$Z X$ and $W Y:$ "... d'un rouge passionné et de reflets intenses".

$Z Y$ and $W X:$ "... d'un rouge intense et de reflets passionnés".

$\rightarrow$ Solution proposée : substantivation de "passionné » et suppression de la coordination (et) :

«... d'un rouge passion aux reflets intenses ».

\section{Exemple d'exploitation didactique}

"Parmi toutes les difficultés que j'ai rencontrées pour traduire ce texte, le point qui a retenu mon attention est celui de la structure new passionate and intense red shades... / le problème le plus ardu dans ce texte me paraît être celui de l'expression to fall head over in love (par exemple). Ainsi, la reformulation de la coordination (...passionate and intense...) ou encore le transfert d'images culturelles (fall head over) pose problème parce qu'elles n'ont pas de contrepartie exacte dans la langue française. À titre d'exemple, le segment initial donne lieu à au moins trois possibilités de reformulation différentes (citer les expres-

(1) Guidère, Introduction à la traductologie. 
sions possibles). La traduction que j'ai proposée de ce segment / de ces mots / de cette expression idiomatique s'inscrit dans une approche cibliste parce qu'elle vise à adapter le sens du texte traduit au public récepteur afin qu'il y ait le même effet de sens à l'arrivée. Ainsi, j'ai traduit telle expression / tel mot... par tel ou tel équivalent parce qu'il correspond mieux à la culture / à l'attente des lecteurs / récepteurs francophones qui sont habitués à tel aspect / qui ne sont pas familiers de tel aspect... Mais j'aurais pu traduire ce mot / cette expression par (citer la proposition non retenue), auquel cas j'aurais été amené à privilégier le point de vue de la source, mais le public cible aurait probablement compris que... etc. ".

\section{Étude de cas 2 : Lancaster Skin Therapy}

\begin{tabular}{|c|c|}
\hline Version anglaise & Version française \\
\hline SKIN THERAPY & SKIN THERAPY \\
\hline VITAL & SOURCE \\
\hline OXYGEN & D'OXYGÈNE \\
\hline SUPPLY FOR & CONTOUR DES \\
\hline EYES & YEUX \\
\hline $\begin{array}{l}\text { - Close your eyes to signs of daily stress } \\
\text { and fatigue. Say goodnight to fine lines } \\
\text { and wrinkles. Now, Lancaster com- } \\
\text { bines the unprecedented benefits of } \\
\text { Skin Therapy Vital Oxygen Supply } \\
\text { with deeply soothing botanicals to crea- } \\
\text { te this highly-targeted, "lighter-than- } \\
\text { air" eye treatment. And the results are } \\
\text { truly eye-opening. }\end{array}$ & $\begin{array}{l}\text { - Fermez les yeux. Finis les éternels } \\
\text { signes de stress et de fatigue. Dites } \\
\text { adieu aux rides et aux ridules ! } \\
\text { Désormais, Lancaster vous offre un } \\
\text { soin ciblé pour le contour des yeux } \\
\text { léger comme une plume, qui associe } \\
\text { les bienfaits sans précédent de Skin } \\
\text { Therapy Source d'Oxygène Vitale aux } \\
\text { vertus apaisantes des extraits de } \\
\text { plantes. Vous n'en croirez pas vos } \\
\text { yeux! }\end{array}$ \\
\hline $\begin{array}{l}\text { - Pure oxygen molecules and natural } \\
\text { extracts infuse the fragile skin around } \\
\text { your eyes with intensive moisture. }\end{array}$ & $\begin{array}{l}\text { - Les molécules d'oxygène* ajoutées } \\
\text { aux extraits naturels se fixent sur les } \\
\text { zones fragiles de l'œil pour une hydra- } \\
\text { tation intense. }\end{array}$ \\
\hline
\end{tabular}




\begin{tabular}{|c|c|}
\hline $\begin{array}{l}\text { Dark circles caused by stress and } \\
\text { fatigue are visibly softened. }\end{array}$ & $\begin{array}{l}- \text { Les cernes dus a } \\
\text { sont visiblemen }\end{array}$ \\
\hline $\begin{array}{l}\text { - Accumulated fluids are dispersed to } \\
\text { relieve under-eye puffiness. }\end{array}$ & $\begin{array}{l}\text { ionne et atté- } \\
\text { sous les yeux. }\end{array}$ \\
\hline $\begin{array}{l}\text { - Skin's own anti-free radical protection } \\
\text { system is reinforced to help diffuse } \\
\text { potential damage. }\end{array}$ & $\begin{array}{l}\text { - Le système de protection naturel de la } \\
\text { peau est renforcé pour éviter les agres- } \\
\text { sions. }\end{array}$ \\
\hline $\begin{array}{l}\text { - Fragile skin's resistance to expression } \\
\text { lines is strengthened. }\end{array}$ & $\begin{array}{l}\text { - La peau fragile du contour des yeux } \\
\text { garde son élasticité et les rides } \\
\text { s'estompent. }\end{array}$ \\
\hline y eye concerns are & $\begin{array}{l}\text { Faites de beaux rêves avec Source } \\
\text { d'Oxygène Contour des Yeux. }\end{array}$ \\
\hline ewed, thoroughly & Pour \\
\hline $\begin{array}{l}\text { netric Oxygen } \mathrm{Ca} \\
\text { endino. }\end{array}$ & $\begin{array}{l}\text { *S.A.T.O. : Système Asymétriqu } \\
\text { Transport d'Oxygène. Brevet en inst }\end{array}$ \\
\hline
\end{tabular}

\section{Justification des équivalences choisies}

Deeply soothing botanicals: dans les dictionnaires consultés, botanicals désignent des médicaments à base de plante ou, plus généralement, des extraits de plantes. Cette dernière formulation s'utilise fréquemment dans le domaine cosmétique et semble donc plus pertinente pour notre traduction. Pour soothing, l'adjectif " apaisant " convient mieux que "calmant ", ce dernier faisant plutôt référence à un médicament. Enfin, pour ce qui est de l'adverbe deeply, il est préférable de l'omettre dans la traduction car il rendrait la construction de la phrase alambiquée. Cependant, l'idée de soothing peut se rendre avec les substantifs "propriété " ou "vertus ", qui permettent de renforcer le pouvoir apaisant qu'aurait le produit. Pour toutes ces raisons, nous proposons comme traduction du segment Deeply soothing botanicals : les vertus apaisantes des extraits de plantes.

Results are truly eye-opening: les recherches sur Internet ont donné les résultats suivants : 


\section{Main Entry: eye-opener}

Function: noun

1: a drink intended to wake one up 2: something startling, surprising, or enlightening: her biography is a real eye-opener.

- eye-opening adjective(2).

On en conclut que surprenant et étonnant seraient des adjectifs qui conviendraient bien en français. Toutefois, mieux vaut préserver le jeu de mots sur l'œil pour trouver une formule à la fois accrocheuse et fidèle au sens en anglais. De plus, n'oublions pas truly qui vient renforcer les résultats supposés du produit. Il faudra donc privilégier des tournures idiomatiques comme :

Vous n'en croirez pas vos yeux ! Les résultats sautent aux yeux !, etc.

Accumulated fluids: ces "liquides accumulés " correspondent à de petites quantités d'eau et de sang qui s'accumulent sous les yeux. Les tissus de la peau retiennent alors ces liquides et les yeux gonflent, formant des poches sous les yeux. Cette notion de "liquides accumulés " est très floue, le lecteur francophone aura besoin d'une explication claire. Comme le support publicitaire requiert des restrictions d'espace, mieux vaut tourner la phrase autrement pour éviter toute ambiguiité. D'ailleurs, la voie passive en anglais serait malheureuse en français, la voie active est donc à privilégier, d'où la traduction par "décongestionne et atténue ».

Skin's own anti-free radical protection: le Grand dictionnaire terminologique(3) définit le terme radical comme suit :

A basic group of atoms which is unchanged during ordinary reaction of the compound of which it is a constituent. Cela correspond bien à radicaux en français (définition du Petit Robert) :

"Groupement d'atomes, présent dans une série de molécules. » Et radicaux libres :

" non combinés à d'autres atomes ou groupement d'atomes. "

(2) (Voir la définition que donne le Merriam-Webster en ligne (www.merriam-webster.com/dictionary) pour eye-opening).

(3) www.granddictionnaire.com 
Voici ce que dit le Grand dictionnaire terminologique à propos des radicaux libres :

Définition :

"État intermédiaire que prend un atome, un ion ou une molécule, qui laisse un électron libre sur son orbite externe, et qui a pour effet d'accroître sa réactivité à l'égard des molécules voisines [...]. Le terme est très souvent employé au pluriel, tant en anglais qu'en français (radicaux libres, free radicals), pour désigner, de façon large, l'ensemble des molécules dérivées du métabolisme de l'oxygène qui peuvent causer des dommages oxydatifs à un organisme. "

On peut donc dire que le phénomène chimique auquel nous avons affaire ici correspondrait en français au "système de protection antiradicalaire de la peau ou système de protection contre les radicaux libres ». Ces deux solutions sont acceptables mais il serait plus judicieux de ne pas introduire de notions trop techniques dans ce texte publicitaire. Certes, ces notions visent sur l'original à témoigner du sérieux et du soubassement scientifique de la marque, mais une technicité excessive pourrait rebuter le consommateur francophone ou le laisser perplexe face au message publicitaire. Il convient par conséquent de privilégier ici l'équivalence « système de protection naturel de la peau".

\section{Commentaire argumenté}

Devant un texte publicitaire comportant autant d'expressions ou de jeux de mots relatifs aux yeux et à la nuit, on peut commencer par faire une liste de tournures françaises sur ces deux thèmes pour ensuite faire un tri et sélectionner celles qui conviendraient dans notre traduction.

Bol d'air frais pour vos yeux - Fermer les yeux sur - Se faire des yeux de biche - Bichonnez vos yeux - Garder un oil sur - Ne pas en croire ses yeux - Fini les yeux pochés - Pour des yeux brillants - Les yeux s'ouvrent sur un jour nouveau, etc. 
Une autre difficulté de ce texte publicitaire se trouve dans le mélange des phrases au contenu technique et des expressions familières qui jouent sur le thème de l'œil. Ainsi, l'on rencontre du vocabulaire qui relève du registre médical comme :

- soothing botanicals

- eye treatment

- accumulated fluids

- anti-free radical protection system

- AOCS

D'un autre côté, nous avons affaire à des expressions de la langue courante, telles que :

- say goodnight to

- fine lines and wrinkles

- undereye puffiness

- eyes greet the day

En français, il faudra privilégier le côté idiomatique de la langue, sans tomber dans le " trop familier " ou le " trop technique ». Ainsi, l'on peut utiliser une ou deux expressions du type Vous n'en croirez pas vos yeux mais il faudra garder la phraséologie que l'on retrouve habituellement dans les publicités pour les cosmétiques, comme les rides sont atténuées, un soin qui décongestionne les poches, le système de protection naturel de la peau, etc.

\section{Étude de cas 3 :}

\section{traduction argumentée de DayWear}

\begin{tabular}{|l|l|}
\hline \multicolumn{1}{|c|}{ Version anglaise } & \multicolumn{1}{c|}{ Version française } \\
\hline $\begin{array}{l}\text { Are you wearing your anti-oxidant UV } \\
\text { protection today? }\end{array}$ & $\begin{array}{l}\text { Vous avez sûrement pensé à votre pro- } \\
\text { tection UV aujourd'hui ! }\end{array}$ \\
Day Wear & DayWear \\
Protective Anti-Oxidant Crème or Lotion \\
SPF 15 & Crème ou lotion protectrice antioxydant \\
SPF 15
\end{tabular}


Only from Estée Lauder Research

Every day, treat your skin to the anti-oxidant, UV protection of DayWear.

This deliciously light moisturiser contains a sunscreen and an exclusive blend of anti-oxidant vitamins, botanical extracts and herbs.

Now you can help prevent the damaging effects of UV light and other harsh environmental factors that can cause premature ageing of the skin - and see better looking skin in the future with DayWear.

ESTÉE LAUDER
Des laboratoires Estée Lauder

Grâce à DayWear, votre peau est protégée chaque jour contre les radicaux libres et les UV.

Ce soin hydratant léger et agréable est doté d'une protection anti-UV et d'un principe actif exclusif.

Vous pouvez désormais éviter les effets néfastes des rayons UV et d'autres agressions externes qui causent un vieillissement prématuré de la peau. Grâce à DayWear, votre peau sera toujours plus belle.

ESTÉE LAUDER

\section{Recherche d'une solution lexicographique}

- Research: recherche, laboratoire, étude.

- Botanical extracts: extraits végétaux, extraits de plantes.

- Herbs: herbes, plantes.

- Blend: mélange, alliance, composition.

\section{Justification des équivalences choisies}

- Le mot research pose problème car en anglais, on parle de " recherche ", alors qu'en français, on emploierait plutôt le concept de "laboratoire " ou de " technologie ". Nous avons finalement choisi « laboratoire" parce qu'il est plus idiomatique.

- Traduit littéralement le segment botanical extracts donne peu de sens à la phrase. Il est nettement préférable de parler ici d' " extraits de plantes " parce qu'il s'agit de produits de beauté, et ce type de composant est fréquent, que ce soit pour un soin du visage ou pour un savon pour le corps. De plus, on peut difficilement parler 
d'" extraits de plantes et d'herbes ", car les herbes sont signifiées par l'hypéronyme " plantes". C'est pourquoi herbs a été omis dans la traduction.

\section{Possibilités de traduction}

- Phrase d'accroche : Are you wearing your anti-oxidant UV protection today?

a) Êtes-vous protégé contre les radicaux libres et les rayons UV aujourd'hui ?

b) Avez-vous pensé à mettre votre protection UV antioxydant aujourd'hui ?

c) Vous portez sûrement votre protection UV antioxydante aujourd'hui !

d) Vous avez sûrement pensé à votre protection UV antioxydant aujourd'hui !

Solution proposée : b (pour conserver la question) et $d$ (pour remplacer par une affirmative). Nous avons finalement opté pour l'affirmative : Vous avez sûrement pensé à votre protection UV aujourd'hui !

- Sous-titre 1 : Protective anti-oxidant creme or lotion SPF 15

a) Crème ou lotion Protective antioxydant SPF 15.

b) Crème ou émulsion protectrice antioxydant SPF 15.

c) Crème et émulsion protection jeunesse SPF 15.

Solution proposée : Crème ou lotion protectrice antioxydant SPF 15.

- Sous-titre 2 : Only from Estée Lauder Research

a) Des laboratoires Estée Lauder.

b) Uniquement par Estée Lauder.

c) Par Estée Lauder.

Solution proposée : des laboratoires Estée Lauder. 
- Phrase 1 : Every day, treat your skin to the anti-oxidant, UV protection of Day Wear.

a) Chaque jour, protégez votre peau contre les radicaux libres et les UV avec DayWear.

b) Grâce à DayWear, votre peau est protégée chaque jour contre les radicaux libres et les UV.

Solution proposée : grâce à DayWear, votre peau est protégée chaque jour contre les radicaux libres et les UV.

- Phrase 2 : This deliciously light moisturiser contains a sunscreen and an exclusive blend of anti-oxidant vitamins, botanical extracts and herbs.

a) Ce soin hydratant léger et délicat est doté d'une protection antiUV et d'un mélange exclusif d'antioxydants et d'extraits de plantes.

b) Ce soin hydratant léger et agréable associe une protection antiUV à un mélange exclusif d'antioxydants et d'extraits de plantes.

Solution proposée : Ce soin hydratant léger et agréable est doté d'une protection anti-UV et d'un mélange exclusif d'antioxydants et d'extraits de plantes.

- Phrase 3 : Now you can help prevent the damaging effects of UV light and other harsh environmental factors that can cause premature ageing of the skin - and see better looking skin in the future with DayWear.

a) Maintenant, vous pouvez éviter les effets néfastes des rayons UV et d'autres facteurs environnementaux causant un vieillissement prématuré de la peau. Grâce à DayWear, votre peau sera toujours plus belle.

b) Désormais, vous pouvez éviter les effets néfastes des rayons UV et d'autres facteurs environnementaux causant un vieillissement prématuré de la peau. Grâce à DayWear, votre peau sera toujours plus belle. 
c) Vous pouvez désormais éviter les effets néfastes des rayons UV et d'autres agressions externes causant un vieillissement prématuré de la peau. Grâce à DayWear, votre peau sera toujours plus belle.

d) Vous pouvez désormais éviter les effets néfastes des rayons UV et d'autres agressions externes causant un vieillissement prématuré de la peau et grâce à DayWear, votre peau sera toujours plus belle.

Solution proposée : Vous pouvez désormais éviter les effets néfastes des rayons UV et d'autres agressions externes causant un vieillissement prématuré de la peau. Grâce à DayWear, votre peau sera toujours plus belle.

\section{Commentaire argumenté}

Le segment final and see better looking skin in the future with Day Wear fait partie de la phrase Now you can help prevent the damaging effects of UV light and other harsh environmental factors that can cause premature ageing of the skin [...]. En français, il est difficile d'établir le même lien syntaxique entre ces deux segments séparés par un tiret. Nous avons préféré isoler le deuxième segment et en faire une phrase. En traduisant la phrase par "Grâce à DayWear, votre peau sera toujours plus belle ", nous faisons d'une pierre deux coups : raccourcir la phrase originale en la scindant en deux et offrir une formulation accrocheuse pour le récepteur francophone. Ainsi, on a pu valoriser le produit en mettant "grâce à DayWear " en début de phrase. Le texte anglais s'adresse directement au consommateur avec la mise en avant du pronom You associé au verbe à l'impératif. En français, nous avons choisi de mettre en relief le produit et ensuite le consommateur.

\section{Conclusion}

L'argumentation traductologique est une question complexe qui ne peut être réduite à la « note du traducteur» $(\mathrm{NdT})$. Elle révèle à la fois des options théoriques (sourcières, ciblistes...) et des choix pratiques 
(sémantiques, stylistiques...) qui débordent le cadre ponctuel des textes à traduire. Même si l'argumentation fait partie intégrante de la réflexion sous-jacente à la traduction, justifier ces options et ces choix s'impose au traducteur dans certains contextes tels que la publicité, la presse ou les médias. En d'autres termes, la traduction argumentée est une exigence partout où les enjeux économiques et/ou politiques de la traduction sont importants.

Mais pratiquer la traduction argumentée relève d'une technique spécifique qui s'inscrit dans une logique de gestion de la qualité. Elle permet, en effet, de minimiser la dimension subjective de l'acte traductionnel en offrant des arguments objectifs à l'appui des décisions langagières du traducteur. Envisager la traduction argumentée comme une consolidation de la qualité contribue également à renforcer l'image de marque de la profession en mettant en avant une compétence d'analyse et de synthèse qui valorise le travail entrepris. C'est aussi sortir de l'idée simpliste que la traduction est un acte simple de transposition. Argumenter le produit final, c'est sortir la traduction de la zone d'ombre qui entoure le processus de construction du discours entre deux langues. En somme, le traducteur est certes un artisan du langage mais c'est un artisan conscient de ses choix et de ses décisions.

Mathieu.Guidere@eti.unige.ch

\section{Bibliographie indicative}

Guidère M. (2008), La Communication multilingue, Paris/Bruxelles, De Boeck, collection « Traducto».

Guidère M., (2007), "Qu'est-ce que la traduction finalisée ? ", in Hieronymus, ASTTI, n 4, 2007, p. 7-13.

Guidère M. (2002), "La traduction publicitaire et ses perspectives d'avenir ", in la revue Hieronymus, Genève, nº 2, 2002, p. 9-14. 
Guidère M. (2001), " Translating Practices in International Advertising ", in Translation Journal, vol. 5, $\mathrm{n}^{\circ} 1,2001$, accessible sur http://accurapid.com/journal/15advert.htm

Guidère M. (2000), "Stratégies publicitaires et Traduction stratégique ", in Humanisme et Entreprise, $n^{\circ} 242,2000$, p. 53-64.

Guidère M. (2000), "Aspects de la traduction publicitaire ", in $B A B E L$, Revue de la Fédération Internationale des Traducteurs (FIT), 2000, vol. 46, $\mathrm{n}^{\circ} 1$, p. 20-40.

Guidère M. (2000), "Sémiotique comparée du territoire : les stratégies territoriales en publicité internationale ", in Territoires sous influence, 2000, p. 120-144.

Guidère M. (2000), Publicité et traduction, Paris, L'Harmattan, collection "Communication en pratique".

Mathieu Guidère est professeur de traductologie à l'université de Genève (ETI) et membre du LIDILEM (Laboratoire de linguistique et didactique des langues étrangères et maternelles), Université Stendhal Grenoble 3.

Il est directeur de la collection Traducto chez De Boeck. http://universite.deboeck.com/collections/?collection_id=241 
Traduire la publicité ou Comment justifier et argumenter ses choix de traduction 
